\title{
Anomalous origin of pulmonary artery branch from aorta ascendens- Outcome of patients who underwent direct re-implantation
}

\author{
Javid Raja ${ }^{1}$, Sabarinath Menon ${ }^{1}$, Arun Gopalakishnan ${ }^{1}$, Suneel P.R ${ }^{1}$, and Baiju Dharan ${ }^{1}$ \\ ${ }^{1}$ Sree Chitra Tirunal Institute for Medical Sciences and Technology
}

January 11, 2021

\begin{abstract}
Background: Anomalous origin of pulmonary artery branch from ascending aorta(AOPA) in the presence of two separate semilunar valves is a rare but important entity necessitating early diagnosis and surgery to prevent development of irreversible pulmonary vascular disease. We evaluated our experience with the technique and outcome of eleven patients with this condition. Methods: Between January 2000 and December 2018, eleven patients were diagnosed with AOPA. Echocardiographic data were collected from the records, regarding the site of origin of the anomalous pulmonary artery, additional defects, biventricular function and pulmonary artery pressures. Intraoperative charts were reviewed for the details of the surgical procedure and cardiopulmonary bypass. Postoperative data included survival, ventilatory support and duration of hospital stay. After discharge, children were reviewed at 1 month, 3 months, 6 months and then at yearly intervals. Results: Of the eleven patients, male to female ratio was $4: 7$ with a median age of six months(15 days - 28 years) and median weight of $5.7 \mathrm{~kg}(1.8 \mathrm{~kg}-40 \mathrm{~kg}) . \mathrm{Nine}$ patients underwent direct re-implantation of anomalous pulmonary artery branch to main pulmonary artery. The survival rate was $91 \%$ in our series. On follow-up, no re-operations or re-interventions were required and all patients who underwent surgical correction were in stable clinical condition. Conclusion: Early and midterm outcomes of children who underwent surgery for AOPA is good. Early direct re-implantation of the anomalous branch PA to main pulmonary artery is the optimal surgical strategy for these patients
\end{abstract}

Title page

Type of article: Original article

Title of the article: Anomalous origin of pulmonary artery branch from aorta ascendens- Outcome of patients who underwent direct re-implantation

Running title: Outcome of Hemitruncus patients

Place of study: Department of Cardiothoracic and vascular surgery

Sree Chitra Tirunal Institute for Medical Sciences \& Technology,

Trivandrum

India - 695011

AUTHORS:

1. Javid Raja, Mch ${ }^{1}$

2. Sabarinath Menon, $\mathrm{Mch}^{2}$ 
3. Arun Gopalakrishnan, $\mathrm{DM}^{3}$

4. Suneel P.R., $\mathrm{DM}^{4}$

5. Baiju S. Dharan, $\mathrm{Mch}^{5}$

Department(s) and institution(s):

$1=$ Associate Consultant, Fortis Escorts Heart Institute, New Delhi

$2=$ Additional Professor, Department of Cardiothoracic and vascular surgery, Sree Chitra Tirunal

Institute for Medical Sciences \& Technology, Trivandrum

$3=$ Assistant professor, Department of Cardiology, Sree Chitra Tirunal Institute for Medical

Sciences \& Technology, Trivandrum

$4=$ Professor, Department of Cardiac anaesthesia, Sree Chitra Tirunal Institute for Medical

Sciences \& Technology, Trivandrum

$5=$ Professor and Head, Department of Cardiothoracic and vascular surgery, Sree Chitra Tirunal

Institute for Medical Sciences \& Technology, Trivandrum

CORRESPONDING AUTHOR:

Dr. Sabarinath Menon

Additional Professor in Pediatric cardiac surgery

Department of Cardiothoracic and vascular surgery

Sree Chitra Tirunal Institute for Medical Sciences \& Technology

Trivandrum - 695011

Telephone: +919995284442

Email: sabarinath.menon@gmail.com

Meeting Name: 20th Annual Conference of Paediatric Cardiac Society of India

City: Raipur, Chhattisharh

Country: India

Date: 10th October 2019

Word Count: 2872 words

Conflicts of Interest: None

Funding: No assistance/financial support taken from anybody for this work

Patient consent: Obtained

\section{ABSTRACT}

Background:

Anomalous origin of pulmonary artery branch from ascending aorta(AOPA) in the presence of two separate semilunar valves is a rare but important entity necessitating early diagnosis and surgery to prevent development of irreversible pulmonary vascular disease. We evaluated our experience with the technique and outcome of eleven patients with this condition. 


\section{Methods:}

Between January 2000 and December 2018, eleven patients were diagnosed with AOPA. Echocardiographic data were collected from the records, regarding the site of origin of the anomalous pulmonary artery, additional defects, biventricular function and pulmonary artery pressures. Intraoperative charts were reviewed for the details of the surgical procedure and cardiopulmonary bypass. Postoperative data included survival, ventilatory support and duration of hospital stay. After discharge, children were reviewed at 1 month, 3 months, 6 months and then at yearly intervals.

Results:

Of the eleven patients, male to female ratio was $4: 7$ with a median age of six months(15 days - 28 years) and median weight of $5.7 \mathrm{~kg}(1.8 \mathrm{~kg}-40 \mathrm{~kg})$. Nine patients underwent direct re-implantation of anomalous pulmonary artery branch to main pulmonary artery. The survival rate was $91 \%$ in our series. On follow-up, no re-operations or re-interventions were required and all patients who underwent surgical correction were in stable clinical condition.

Conclusion:

Early and midterm outcomes of children who underwent surgery for AOPA is good. Early direct reimplantation of the anomalous branch PA to main pulmonary artery is the optimal surgical strategy for these patients.

Key Words: Anomalous origin of pulmonary artery branch from ascending aorta, Direct re-implantation, Pulmonary artery hyperension

\section{ABBREVIATIONS:}

AOPA - Anomalous origin of one branch of pulmonary artery from the aorta

PAH- Pulmonary artery hypertension

PA - Pulmonary artery

RPA- Right pulmonary artery,

LPA- Left Pulmonary artery

SAM - Subaortic membrane

CPB - Cardiopulmonary bypass

MPAP - Mean pulmonary artery pressure

ICU - Intensive care unit

RVSP - Right Ventricle Systolic Pressure

PDA- Patent Ductus Arteriosus,

ICR- Intracardiac Repair,

AXC - Aortic Cross clamp time,

SSI - Surgical Site Infection

Introduction:

Anomalous origin of one pulmonary artery branch from the ascending aorta(AOPA) is a rare congenital cardiovascular anomaly accounting for less than $0.1 \%$ of congenital heart defects.[1] The term "Hemitruncus" which was used previously for this condition is seldom used now because of the presence of two separate semilunar valves in this condition. AOPA is classified depending on the site of origin as, those arising proximally from the posterior or left posterior aspect of the ascending aorta near the aortic valve, as the 
proximal type and those originating from the ascending aorta just proximal to the innominate artery, as the distal type[3].

Pathophysiologically, AOPA is characterised by early development of pulmonary vascular disease in both lungs. The lung connected to the normally arising PA receives the entire cardiac output from the right ventricle, while the other lung supplied by AOPA is exposed to both volume and pressure overload due to unrestricted shunting from the aorta. This leads to early development of pulmonary vascular occlusive disease, the mechanisms of which include circulating vasoconstrictor substances, neurogenic crossover from the unprotected lung to the protected one and development of pulmonary hypertension secondary to left ventricular failure [5]. In this study we evaluated the outcome of patients with this rare condition and the challenges in the peri-operative management of this condition.

Material and Methods:

A retrospective analysis of all patients who were diagnosed with AOPA was performed after getting approval from our institutional Ethical committee. Between January 2000 and December 2018, eleven patients were diagnosed to have AOPA of which nine children underwent surgical correction. Detailed information of these patients is shown in Table 1 and Table 2.

All the patients presented with features of congestive heart failure except two, who presented late after infancy (one at 4 years and other at 28 years). Eight patients had anomalous origin of right PA(Figure 1) and three had left PA. Associated anomalies include patent ductus arteriosus (7 children), tetralogy of Fallot ( 3 children) and subaortic membrane (SAM) was seen in one. All children except one(patient 6) had left aortic arch(table 1). Diagnosis was established according to the echocardiographic, computed tomography and operative findings. The surgical approach in all cases was via median sternotomy. The anomalous pulmonary artery originating from aorta ascendens, main PA and its branch were carefully mobilized upto the hilum. Cardiopulmonary bypass (CPB) was instituted after high ascending aortic cannulation and bicaval cannulations. The anomalous pulmonary artery was snugged at the initiation of CPB. PDA was ligated and divided in all cases. Mild hypothermic antegrade cardioplegic arrest was used in seven patients. Ascending aorta was transected above the AOPA (Figure 2) and the pulmonary artery branch was harvested with a cuff of posterior wall from the aorta (Figure 3). The defect in the aorta was closed with bovine pericardial patch. The anomalous pulmonary artery was then anastomosed end to side with the lateral aspect of the main pulmonary artery (Figure 4) under direct vision. Two patients underwent re-implantation without cardioplegic arrest. In these patients, a side-biting clamp was applied on the ascending aorta and the anomalous PA was harvested.

Associated procedures include PDA ligation and division in seven patients, intracardiac repair with conduit for one patient with tetralogy of Fallot, SAM excision in one patient and De vega's tricuspid repair in one patient.

Results:

There was one in-hospital mortality $(9 \%)$ in our series(patient no. 9). This child expired in the postoperative period due to severe right ventricular(RV) dysfunction refractory to all medications. This child presented with features of heart failure, was intubated and had severe trumped regurgitation with severe RV dysfunction preoperatively. Child was taken up for surgical repair after medical stabilisation. However child had a stormy postoperative period with severe RV dysfunction and succumbed to death.

All patients received elective inotropic support in the postoperative period. Oral sildenafil was administered to all the children in the immediate postoperative period. Two children developed pulmonary arterial hypertension crisis on the first postoperative day which was managed successfully with pulmonary vasodilators and were extubated after 48 hours. One patient had superficial surgical site infection which was managed with sensitive antibiotics.

One of the two patients who were not operated had irreversible pulmonary vascular disease (patient 11). This patient was diagnosed to have AOPA in infancy and was advised surgery but was lost to followup 
and presented in adulthood with hemoptysis and succumbed to death at an age of 28 years. Other child (patient 10) underwent cardiac catheterisation at the age of 4 years which showed severe pulmonary arterial hypertension in the left lung that was not reversible with $100 \%$ oxygen and hence was not offered surgical correction as decided in our joint cardiac meet.

The mean ventilatory time and intensive care unit(ICU) stay are shown in Table 1. All patients underwent postoperative echocardiography in hospital and mean pulmonary artery pressure (MPAP) decreased significantly and were discharged in good condition with clinical symptoms relieved.

Followup for all the eight patients ranges from 12 - 78 months. All children are alive, asymptomatic, and in stable clinical condition at the most recent follow-up. Ross modified score [17] was used to assess cardiac function and all patients who underwent surgical correction had scores less than three as shown in Table 3. Systemic saturation of $100 \%$ was measured in all patients. In the recent followup echo, all patients had normal ventricular function with no significant tricuspid regurgitation with Right Ventricle Systolic Pressure(RVSP) of less than 20 in all the patients. Since there was no cardiac catheterisation done in the followup period, the exact PA pressures couldnot be assessed, which is the main limitation of this study.

The doppler flow velocity at the anastomotic site of anomalous pulmonary artery and main pulmonary artery showed no significant gradients(Figure 5) except for one patient(No. 8) who had mild pulmonary stenosis with a peak gradient of $26 \mathrm{mmHg}$ [Table 2]. Child with SAM developed recurrent left ventricular outflow tract obstruction and is awaiting surgery.

\section{COMMENT:}

AOPA from the aorta remains an investigational field in paediatric cardiac surgery due to the low incidence of this congenital malformation $[1,2]$. The largest reported series includes only 16 patients who underwent surgery for this anomaly over a 36 -year period[3]. In our study, the most commonly associated anomaly was PDA, as consistent with a previous report [4].

It has been reported that histological features of pulmonary vascular disease can be seen as early as the first month of life with this condition[14]. One-year survival of children with AOPA is around $30 \%$ without surgical treatment[5]. To avoid developing irreversible pulmonary vascular disease and improve the outcome, AOPA must be repaired in early infancy. All our surviving patients were operated during infancy $[5,6]$.

The first successful surgical correction of AOPA was reported in 1961[9] following which various surgical techniques have been proposed for correction of this anomaly. These include implantation using an autologous pericardial patch, interposition graft or homograft and direct implantation of the anomalous branch to main pulmonary artery[10 - 12]. Considering the growth potential of the pulmonary artery and the prevention of anastomotic obstruction, the direct re-implantation seems to be the procedure of choice provided, it can be achieved without tension [11, 14].

Direct re-implantation after excision of the anomalous PA as a button akin to coronary button in an arterial switch operation, is our technique of choice now. Mobilisation of main pulmonary artery and both branches upto the hilum helps to reduce the tension in the anastomosis. Reconstruction of ascending aorta after the re-implantation (in case of RPA) provides excellent exposure to avoid any inadvertent twists. Reconstruction of harvest site with a pericardial patch reduces the tension in the aortic suture line, thus preventing any posterior displacement leading to compression of translocated RPA.

Patients with AOPA who underwent surgical treatment early in infancy have excellent short and long-term outcome[15]. However, the most common late complication after surgical treatment is the anastomotic site stenosis. Significant stenosis requiring further intervention is considered as an important cause of morbidity[14]. There was no anastomotic site stenosis seen in any of our patients.

The calculation of Pulmonary vascular resistance could be fallacious in AOPA. The source of pulmonary blood flow to both lungs are separate and the pulmonary vascular resistance in each lung could also be different. Hence the net Qp (Pulmonary blood flow) in this situation would be difficult to estimate and 
saturation alone can not be relied for PVR estimation. The ideal scenario, in this situation would be to do a functional MRI to calculate the pulmonary venous return of individual left and right pulmonary veins to calculate the true Qp (Pulmonary blood flow). This would help in calculation the true PVRi in each lung. Hence in this situation if the PVRI is calculated by assuming the Qp, this may result in fallaciously high value[16]. In this series, presence of sub-systemic PA pressures in the AOPA with clinical, X-ray and echocardiographic evidence of operability has been considered for surgical repair. The cut-off for operability based on calculated PVRi is still unknown.

Conclusion:

AOPA is a potentially treatable lesion with an acceptable long-term outcome and near-normal quality of life if operated before infancy. Early direct pulmonary re-implantation is the preferred surgical strategy for this condition as proven by our results. However large number of patients are needed to compare with other surgical techniques to statistically prove it.

\section{REFERENCES:}

Kutsche LM, Van Mierop LH. Anomalous origin of a pulmonary artery from the ascending aorta: associated anomalies and pathogenesis. Am J Cardiol. 1988;61(10):850-856. doi: 10.1016/0002-9149(88)91078-8. [PubMed] [CrossRef] [Google Scholar]

2. Patel RJ, Zakir RM, Sethi V, Patel JN, Apovian J, Alexander JC, et al. Unrepaired tetralogy of fallot with right hemitruncus in an adult: a rare case. Tex Heart Inst J. 2007;34(2):250-251.

[PMC free article] [PubMed] [Google Scholar]

3. Abu-Sulaiman RM, Hashmi A, McCrindle BW, Williams WG, Freedom RM. Anomalous origin of one pulmonary artery from the ascending aorta: 36 years' experience from one centre. Cardiol Young. 1998;8(4):449-454. doi: 10.1017/S1047951100007101. [PubMed] [CrossRef]

[Google Scholar]

4. Kajihara N, Imoto Y, Sakamoto M, Ochiai Y, Kan-o M, Joo K, et al. Surgical results of anomalous origin of the right pulmonary artery from the ascending aorta including reoperation for infrequent complications. Ann Thorac Surg.2008;85(4):1407-1411. doi: 10.1016/j.athoracsur. 2007.11.081. [PubMed] [CrossRef] [Google Scholar]

5. Keane JF, Maltz D, Bernhard WF, Corwin RD, Nadas AS. Anomalous origin of one pulmonary artery from the ascending aorta. Diagnostic, physiological and surgical considerations.

Circulation. 1974;50(3):588-594. doi: 10.1161/01.CIR. 50.3.588. [PubMed] [CrossRef] [Google Scholar]

6. Benatar A, Kinsley RH, Milner S, Dansky R, Hummel DA, Levin SE. SurgicaL correction for one pulmonary artery arising from ascending aorta-report of five cases. Int J Cardiol.

1987;16(3): 249-255. doi: 10.1016/0167-5273(87)90148-3. [PubMed] [CrossRef]

\section{[Google Scholar]}

7. Penkoske PA, Castaneda AR, Fyler DC, Van Praagh R. Origin of pulmonary artery branch from ascending aorta. Primary surgical repair in infancy. J Thorac Cardiovasc Surg. 1983;85(4):537-

545. [PubMed] [Google Scholar] 
8. Prifti E, Bonacchi M, Murzi B, Crucean A, Leacche M, Bernabei M, et al. Anomalous origin of the right pulmonary artery from the ascending aorta. J Card Surg. 2004;19(2):103-112. doi: 10.1111/j.0886-0440.2004.04023.x. [PubMed][CrossRef] [Google Scholar]

9. Armer RM, Shumacker HB, Klatte EC. Origin of the right pulmonary artery from the ascending aorta. Report of a surgically corrected case. Circulation.1961;24:662-668. doi: 10.1161/01.CIR.

24.3.662. [PubMed] [CrossRef] [Google Scholar]

10. Fucci C, di Carlo DC, Di Donato R, Marino B, Calcaterra G, Marcelletti C. Anomalous origin of the right pulmonary artery from the ascending aorta: repair without cardiopulmonary bypass. Int J Cardiol. 1989;23(3):309-313. doi:10.1016/0167-5273(89)90189-7. [PubMed] [CrossRef] [Google Scholar]

11. Nakamura Y, Yasui H, Kado H, Yonenaga K, Shiokawa Y, Tokunaga S. Anomalous origin of the right pulmonary artery from the ascending aorta. Ann Thorac Surg. 1991;52(6):1285-1291. doi. 10.1016/0003-4975(91)90014-H. [PubMed] [CrossRef] [Google Scholar]

12. Salaymeh KJ, Kimball TR, Manning PB. Anomalous pulmonary artery from the aorta via a patent ductus arteriosus: repair in a premature infant. Ann Thorac Surg. 2000;69(4):1259-1261. doi: 10.1016/S0003-4975(99)01428-9. [PubMed] [CrossRef] [Google Scholar] 13. Peng EW, Shanmugam G, Macarthur KJ, Pollock JC. Ascending aortic origin of a branch pulmonary artery-surgical management and long-term outcome. Eur J Cardiothorac Surg. 2004;26(4):762-766. doi: 10.1016/j.ejcts.2004.07.007. [PubMed] [CrossRef] [Google Scholar] 14. Fong LV, Anderson RH, Siewers RD, Trento A, Park SC. Anomalous origin of one pulmonary artery from the ascending aorta: a review of echocardiographic, catheter, and morphological features. Br Heart J. 1989;62(5):389-395. doi: 10.1136/hrt.62.5.389. [PMC free article] [PubMed] [CrossRef] [Google Scholar]

15. Liu Y, Cheng L, Qian X, Zhu H, Duan W, Yu S, et al. Surgical Correction of Anomalous Origin of One Pulmonary Artery Without Grafts in Infants. J Cardiac Surg. 2015;30(1):85-91.

[PubMed]

16. Dimopoulos K, Diller GP, Opotowsky AR, D'Alto M, Gu H, Giannakoulas G, Budts W, Broberg CS, Veldtman G, Swan L, Beghetti M, Gatzoulis MA. Definition and Management of Segmental Pulmonary Hypertension. J Am Heart Assoc. 2018 Jul 4;7(14):e008587. doi: 10.1161/ JAHA.118.008587. PMID: 29973393; PMCID: PMC6064837.

17. Ross RD, Bollinger RO, Pinsky WW. Grading the severity of congestive heart failure in infants. Pediatr Cardiol. 1992;13(2):72-75. doi: 10.1007/BF00798207. [PubMed] [CrossRef] [Google Scholar] 


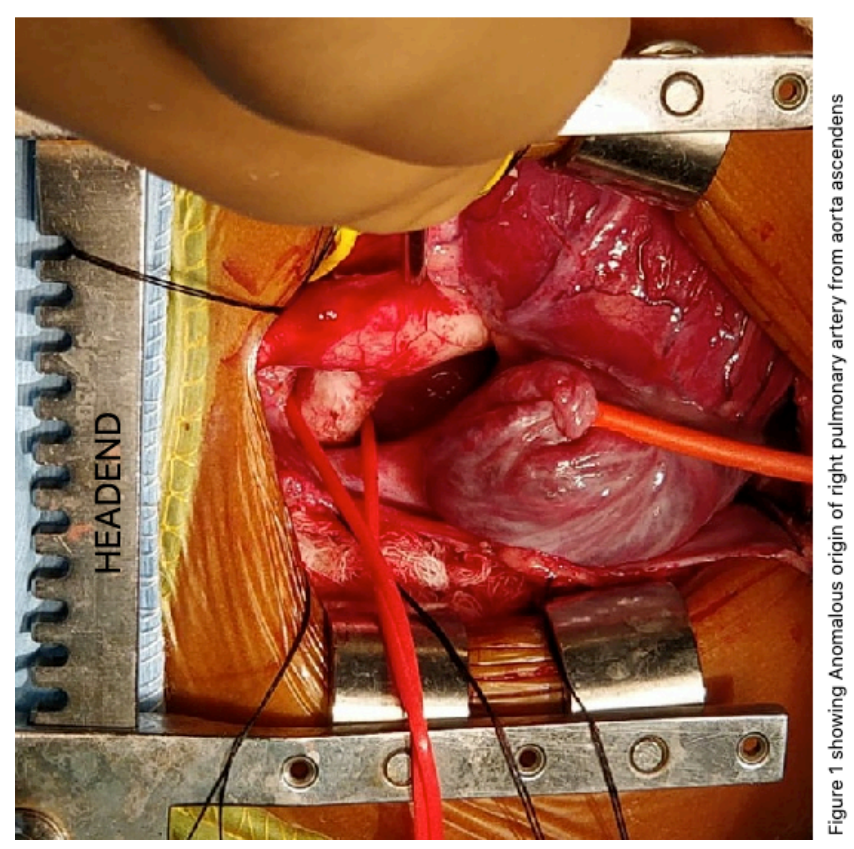




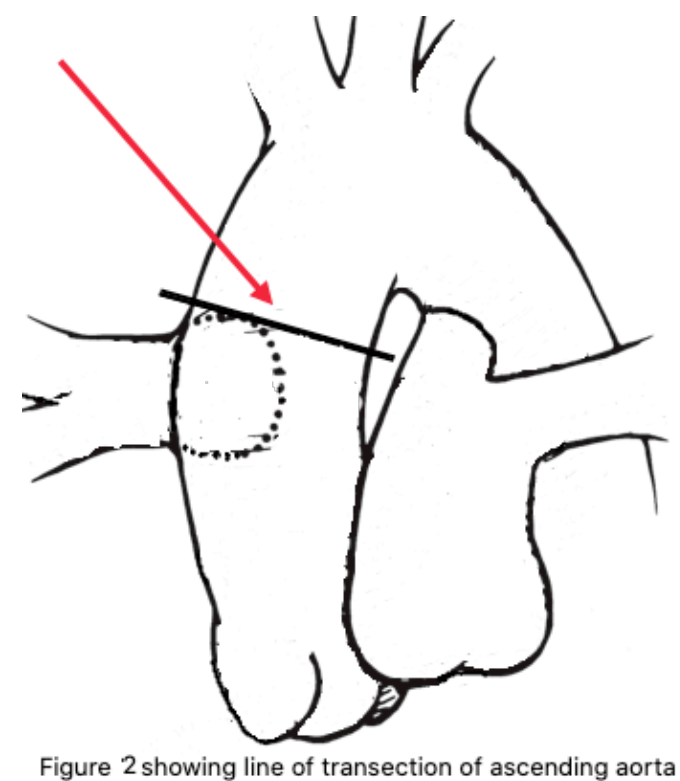

Paint $X$ tite 


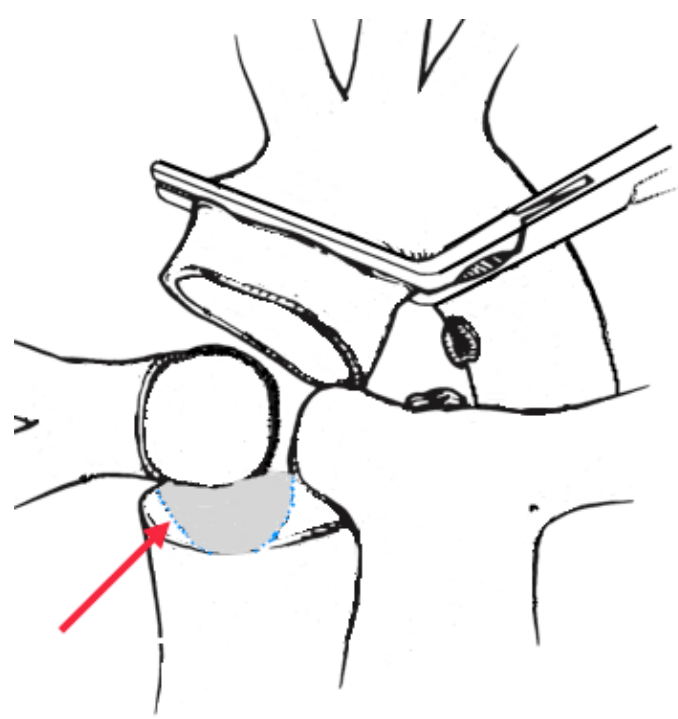

Figure 3 showing the defect in the ascending aorta closed with bovine pericardial patch 


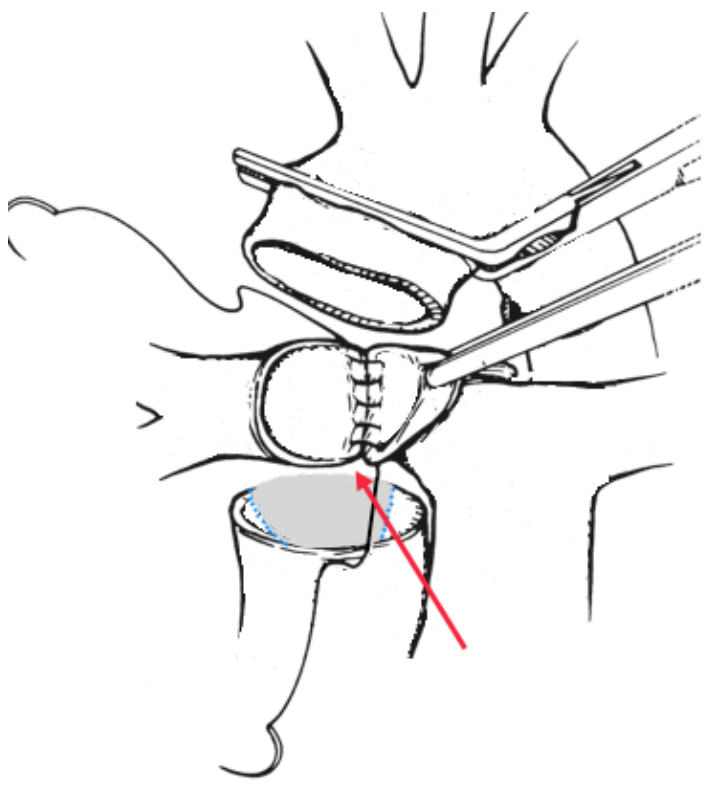

Figure 4 showing Direct re-implantation of right pulmonary artery to ascending aorta 

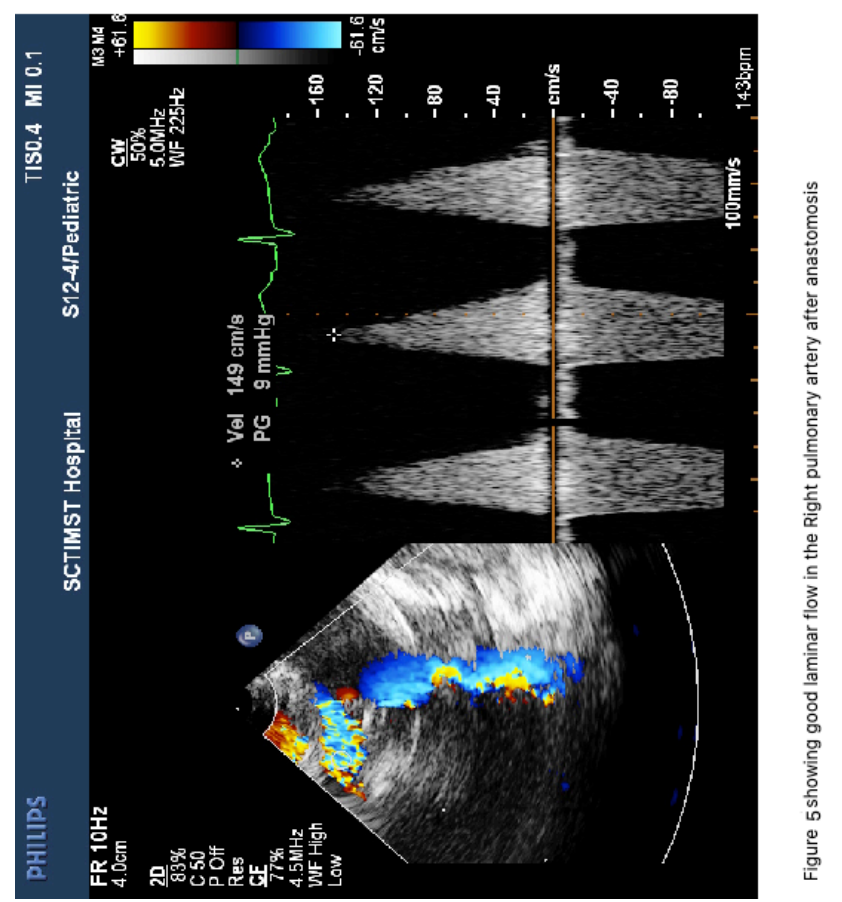

\section{Hosted file}

Table 1 Hemi.pdf available at https://authorea.com/users/324378/articles/503498-anomalousorigin-of-pulmonary-artery-branch-from-aorta-ascendens-outcome-of-patients-whounderwent-direct-re-implantation 
Table 2

\begin{tabular}{|c|c|c|}
\hline PATIENT & $\mathbf{1 0}$ & $\mathbf{1 1}$ \\
\hline AGE (YEARS) & 4 & 28 \\
\hline SEX & M & F \\
\hline WEIGHT $($ KG) & 15 & 40 \\
\hline CHF & ABSENT & ABSENT \\
\hline PRESENTATION & DYSPNOEA ON EXERTION & HEMOPTYSIS \\
\hline PA PRESSURE & $105 / 44$ & $106 / 52$ \\
\hline (mmHg) & & LPA \\
\hline ANOMALOUS BRANCH & LPA & LEFT \\
\hline ARCH & LEFT & TOF \\
\hline OTHER ANOMALIES & TOF & $77 \%$ \\
\hline PREOP SATURATION & $80 \%$ & \\
\hline
\end{tabular}

Table 2 showing Details of patients who were not operated 
Table 3

\begin{tabular}{|c|r|r|r|r|r|r|r|r|r|}
\hline PATIENT & 1 & 2 & 3 & 4 & 5 & 6 & 7 & 8 & 9 \\
\hline ROSS MODIFIED & 1 & 1 & 0 & 2 & 1 & 2 & 0 & 1 & 1 \\
\hline SCORE & & & & & & & & & \\
\hline RVSP & 15 & 13 & 16 & 16 & 17 & 15 & 15 & 17 & 13 \\
\hline PRESSURE GRADIENT & 16 & 18 & 15 & 18 & 14 & 18 & 26 & 16 & 16 \\
\hline (ANASTOMOSIS) & & & & & & & & & \\
\hline LV FUNCTION & $65 \%$ & $70 \%$ & $62 \%$ & $66 \%$ & $65 \%$ & $70 \%$ & $62 \%$ & $66 \%$ & $65 \%$ \\
\hline FOLLOWUP & 78 & 66 & 62 & 55 & 52 & 30 & 14 & 12 & 8 \\
\hline (MONTHS) & & & & & & & & & \\
\hline
\end{tabular}

Table 3 Showing Follow-up Echocardiographic details 